\title{
Generative Design of a Mechanical Pedal
}

\author{
M. FENOON ${ }^{1}$, O. ALQUABEH ${ }^{2}$, M.M. NISAR ${ }^{3}$, S. ZIA ${ }^{4}$. \\ 1University of Debrecen, Faculty of Engineering, Department of Mechanical Engineering, fannoun.mahmoud@gmail.com \\ 2University of Debrecen, Faculty of Engineering, Department of Mechanical Engineering, omeralqawabeh@yahoo.com \\ 3University of Debrecen, Faculty of Engineering, Department of Mechanical Engineering. musanisar93@gmail.com \\ 4University of Debrecen, Faculty of Engineering, Department of Mechanical Engineering, tosalmanzia1995@gmail.com
}

\begin{abstract}
Nowadays, there are various tools that support the initial stages of design available to use for engineers, the traditional Computer-Aided Design (CAD) has been implemented in the engineering components design and replaced manual drafting. However, with the advances and the rapid technology development, new trends emerged to cope with this evolution, namely, Generative Design, Topology Optimization, and Generative Engineering Design. The method is based on numerical algorithms that generate a variety of design and modelling options based on the criteria and constraints set by the designer to allow further design exploration. Proposed in this paper is an implementation of the generative design of a mechanical pedal with further finite element analysis.
\end{abstract}

Keywords: Computer-Aided Design (CAD), Generative Design (GD), Design Optimization, Generative CAD, Rapid Modelling, 3D Modelling, Finite Element Analysis (FEA), Mesh Analysis, Mesh Sensitivity Analyses.

\section{Introduction}

The computer emerged to be a powerful tool utilized in engineering components design with the capability to perform complex 3D modelling and analysis of various shapes and to overcome the manual drawing and drafting. Acknowledging this potential, CAD is currently not only employed in final product design, rather, in the early phases of design as well [1]. Generative CAD supports the users in conceptual design and product development by allowing them to explore a wide range of feasible design options based on the user-defined constraints, components function, aesthetic, performance, and cost [2]. The designing phase has an important role in the cumulative cost, 60 to $80 \%$ of the total cost is committed in the initial stages of conception and design, therefore, efforts have been made to prevent the accumulation of additional costs in design and later on phases [3]. With the use of special algorithms, it allows engineers to optimize their designs around the specific requirement needed for that design, mass reduction is a great example of that, wherein some cases engineers were able to reach up to $45 \%$ lighter product as compared to the design made with the traditional design methods, which greatly benefit the performance in many applications, in drones, cars, and aircraft, where all of these examples can benefit from the lower weight which directly impacts their energy consumption, and fewer materials mean less initial production cost [4]. Furthermore, the development of Additive Manufacturing (AM) technologies, 3D printing in particular, tremendously helped the utilisation of generative design, because manufacturing is no longer limited to the traditional manufacturing process but rather engineers have 
the possibility to design parts that were not possible to manufacture in the past [4]. Several research studies on generative design were made when it first emerged, and most of them were theoretical without real-world applications, then the concept became more feasible and was applied in several applications [5]. Vlah et al implemented the generative design and topology optimization on a race car rocker, with several results of different masses and geometries were generated [5], Gavacova et al made a study on the automotive hood and employed the generative design theory in their work taken into consideration a set of constraints [6], further work in the automotive industry utilizing the generative engineering design was made particularly, in the surface-based components, Gulanová et al conducted a development study in the automotive surface-based components putting into consideration several criteria such as economic, safety, and Aesthetical criteria [7]. In terms of mass reduction, as generative engineering design is oriented on reducing the mass while not compromising the functionality or the effectiveness of the component, a GD study was conducted on an E-bike for reducing its mass while keeping up with the safety requirements, the results show a mass reduction of the frame by $23.913 \%$ [8], another study aiming to reduce the weight while maintaining or preventing strength reduction on a connecting rod of a V8 engine was made with results of $8.13 \%$ of mass reduction without a reduction in the strength [9]. Generative design is becoming widely implemented in all engineering aspects, GD involves both topology optimization and evolutionary scheme, it also simplified the trade-off among the engineering components costs, weights, and performance, the concept was introduced and being utilized in the aviation industry for the purpose of reducing the aircraft weights, enhance their performance, and to reduce the consumption of fuel [10]. Khan et al implemented the concept of GD in generating several yacht hull using GenYacht system which includes a generative design method, the hull is designed according to certain criteria such performance and appearance [11]. As advancements are being made in the field of machine learning, the machine learning techniques are being implemented in designing optimized automotive parts by incorporating the all technical characteristics of the machines. Support Vector Machine (SVM) and nonlinear finite element analysis has been used to make iterative design combinations depending on design parameters to design automotive rubber jounce [12]. Similarly, shape optimization of an automotive rubber bumper was done by introducing a twodimensional optimization problem and using a surrogate model based method to select parameters for solution of optimization problem [13]. With advancement of the artificial intelligence and machine learning more and more studies are implementing these technologies to support the innovation in the design of mechanical parts [14-16]. We can conclusively say, with the current computational capacity and technology, generative engineering design is being employed in different engineering aspects and applications, in this paper a study is conducted on a mechanical pedal aiming to reduce its weight by exploring the alternative design options generated by the GD algorithm.

\section{Methods and materials}

In this study we used generative design to redesign an automotive mechanical pedal, which is a mechanism that controls the throttle valve of the engine, this in turn helps to vary the engine power. The control of the throttle vale by the mechanical pedal influences the volume of air or air fuel mixture into the combustion chamber of an internal combustion engine. Therefore, the weight reduction of 
major automotive components, such as the pedals is so important in automotive design. Normally this part is made by welding 4-5 parts together and is heavy. We intend to modify this part to be 3D printed as a single part by keeping the structural integrity and the strength using less material with the help of generative design. Using solid edge, we created a basic model of the main parts of the pedal assembly which consists of, a pedal, shock absorber that is attached to the pedal and a base using a fixed joint. The pedal base holds the parts together and is attached to car body, the basic $3 \mathrm{~d}$ model of the pedal assembly is shown in Figure 1.

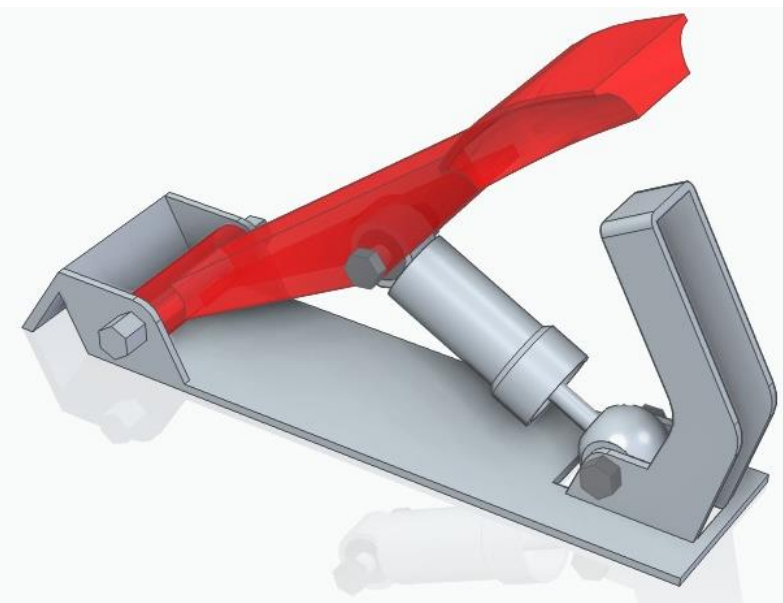

Figure 1. The CAD assembly of the gas pedal designed on Solid Edge

\subsection{Materials}

When choosing the material for the gas pedal, we ensured that the factor of safety is within the allowable limit and give us a room for material reduction and room for design modification, after exploring the engineering materials that are used in the industry we choose (AL- 5050) which has the following mechanical properties:

\begin{tabular}{|c|c|c|c|c|}
\hline \multicolumn{5}{|c|}{ Aluminum 5050 Mechanical Properties } \\
\hline Density & Modulus of Elasticity & $\begin{array}{c}\text { Poisson's } \\
\text { Ratio }\end{array}$ & Yield Stress & Ultimate Stress \\
\hline $2684.000 \mathrm{~kg} / \mathrm{m}^{3}$ & $68947.570 \mathrm{MPa}$ & 0.330 & $55.158 \mathrm{MPa}$ & 144.790 MPa \\
\hline
\end{tabular}

Table 1. Mechanical properties of Al-5050

Based on a study called, "Pedal force determination with respect to ride comfort", they determined that the average force applied to the pedal from the car driver is $450 \mathrm{~N}$ [17]. By Using this value and defining the boundary conditions and using Al-5050 to make an initial Finite Element Analysis for the initial pedal model with a total mass of $0.773 \mathrm{~kg}$, the maximum stress value was $21.7 \mathrm{MPa}$, which makes the factor of safety equal 2.54. This value of factor of safety will allow us to make further modifications to the design using Generative design. 


\subsection{Methods}

Solid Edge 2021 has been used to simulate and optimize the Pedal Design by setting weight as the design variable by keeping the maximum stress under the set limit. Solid Edge 2021 design module allows to simulate the part by setting the material and boundary conditions and then subjecting the part to intended loads. The module can be used for linear static, heat transfer and transient studies. The parameters can be varied and desired results under allowable error percentage and stress values can be achieved. The methods used in simulating the Pedal in Solid Edge environment includes below steps.

- Setting the material to Aluminium 5050 with Yield Stress of $55.158 \mathrm{MPa}$.

- Defining the 2 assembly points of pedal as constraints.

- Applying a load of $450 N$.

- Setting the mesh size depending on the desired accuracy and computational power.

- Solving the model and obtaining the values of stresses and displacement.

Figure 2 shows the set of constraints and loads that were applied and the meshing process carried out after that.
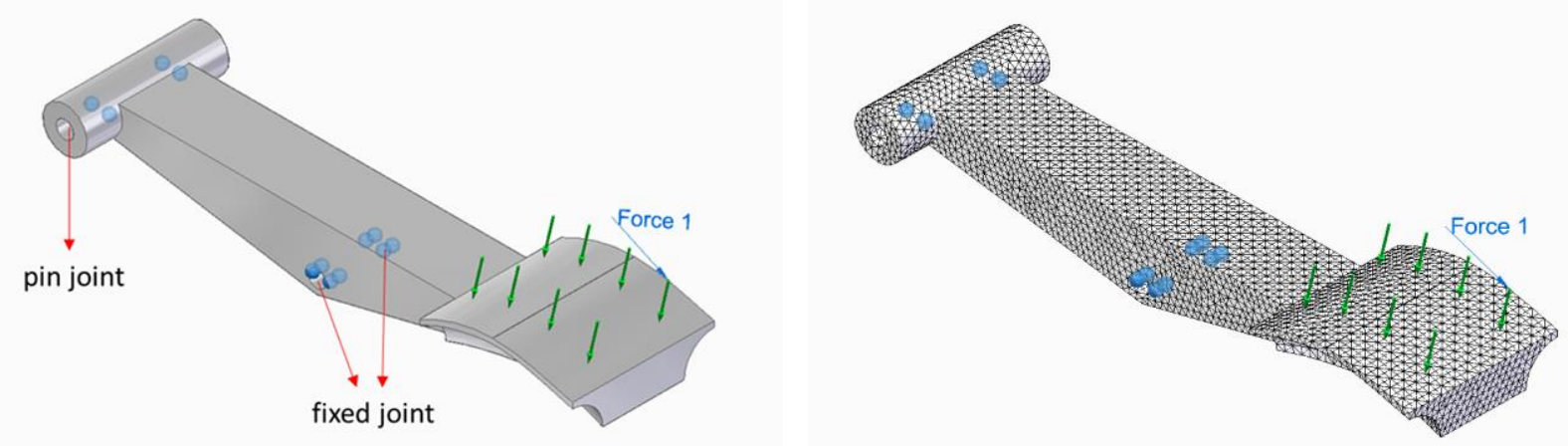

Figure 2. Constraints and loads application (Left) and subsequent mesh process (Right)

After successful simulation run in Solid Edge 2021, the next phase was to use the generative design module for weight reduction of the Pedal. Generative design module can be used to optimize for a specific value of the model depending on its operating conditions. The mass reduction can be based on a set percentage mass reduction, by adjusting the study quality or by a specific factor of safety. The generative design module provides a real time environment to adjust the design parameters and running the design iterations. The manufacturing methods requirements can also be set and material distribution along the model can also be defined.

The generative design process starts by defining the material for the model with the suitable mechanical properties. The areas that need to be preserved can be specified and the fixed or pin supports can be set, and load can be specified with the offset values. The offset is added to set a limit to material removal depending on the study quality and operational conditions of the part. The mass reduction of the Pedal in Solid Edge 2021 Generative Design Module was done as per below steps. 
- Setting the material to Aluminium 5050 with Yield Stress of 55.158 MPa.

- Setting the sides of the pedal face as preserved areas with $2 \mathrm{~mm}$ offset.

- Setting the 2 assembly points of the pedal as the fixed constraints with $5 \mathrm{~mm}$ offset.

- Applying the force of $450 \mathrm{~N}$ on Pedal face.

- Setting the study quality to a set desired value depending on the computational time.

- Defining the percentage mass reduction as $40 \%$.

- Defining the factor of safety of 1.5 to ensure the structural integrity of the Part and to limit maximum stresses to be within the limit of 55.158Mpa.

\section{Results and Discussions}

The first step for analysing the results was to conduct a mesh sensitivity analysis of the pedal model. The mesh sensitivity analysis involved the measurement of the computational time for each mesh sizes. Furthermore, to measure the accuracy of each mesh size the percentage error between the current maximum stress value and the highest maximum stress value was calculated.

These results have been summarised in the Figure 3 which consists of the computational time versus average mesh size plot and percentage error versus average mesh size plot.

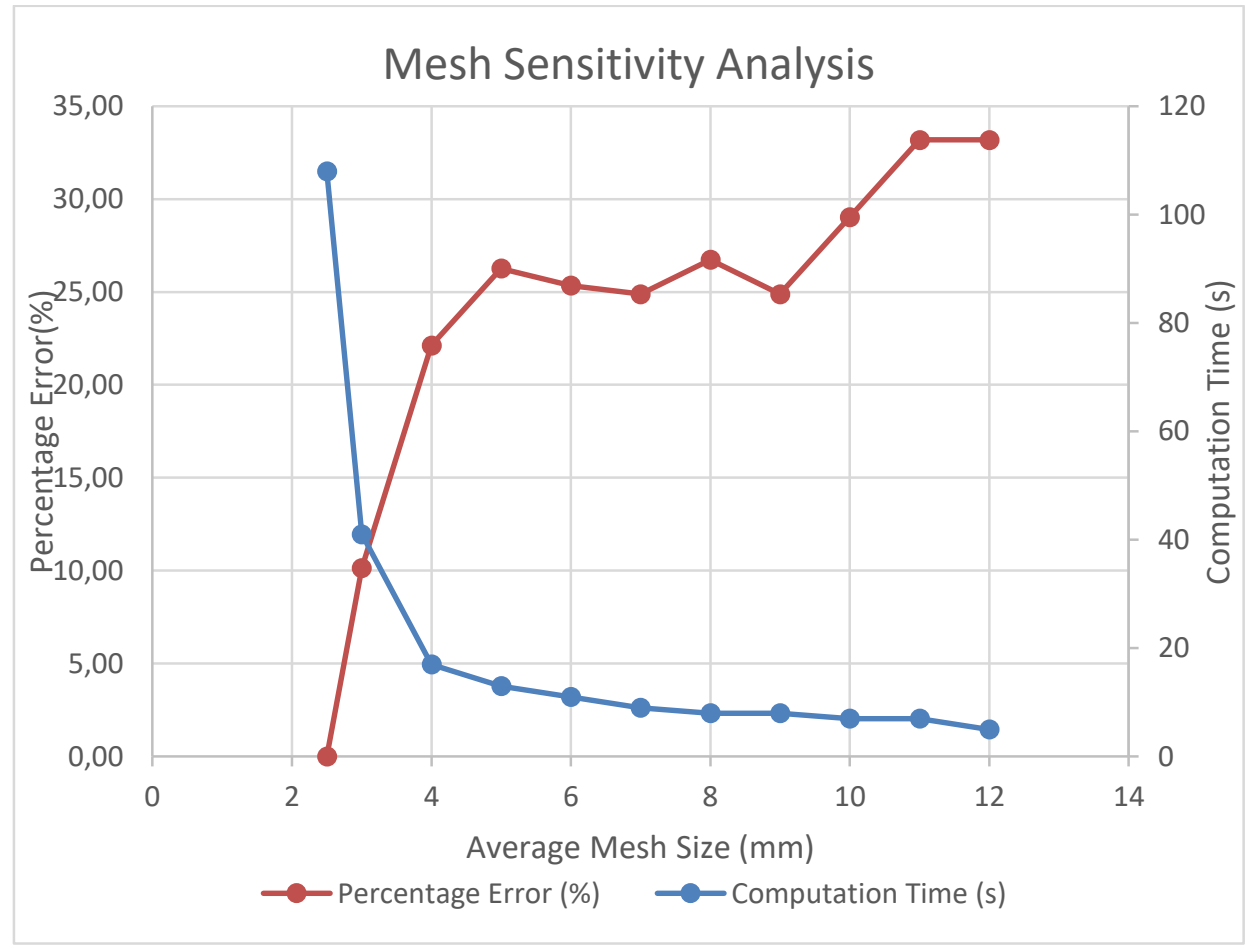

Figure 3. Computation Time versus Average Mesh Size and Percentage Error versus Average Mesh Size

The objective of the mesh sensitivity analysis was to identify the optimum mesh size that could yield the most accurate results of the finite element analysis and consume an appropriate amount of 
computational time. In this case the best result was given by a mesh of element size $2.5 \mathrm{~mm}$ as the computation time was manageable and the accuracy of the result was ideal.

The second step was to conduct the finite element analysis of the designed model and ensure if the maximum stress is in the allowable region considering the material (Aluminium 5050 with Yield Stress of $55.158 \mathrm{MPa}$ ) and an appropriate factor of safety. The results in the form of stress distribution by Von Mises Method and displacement distribution are shown below.
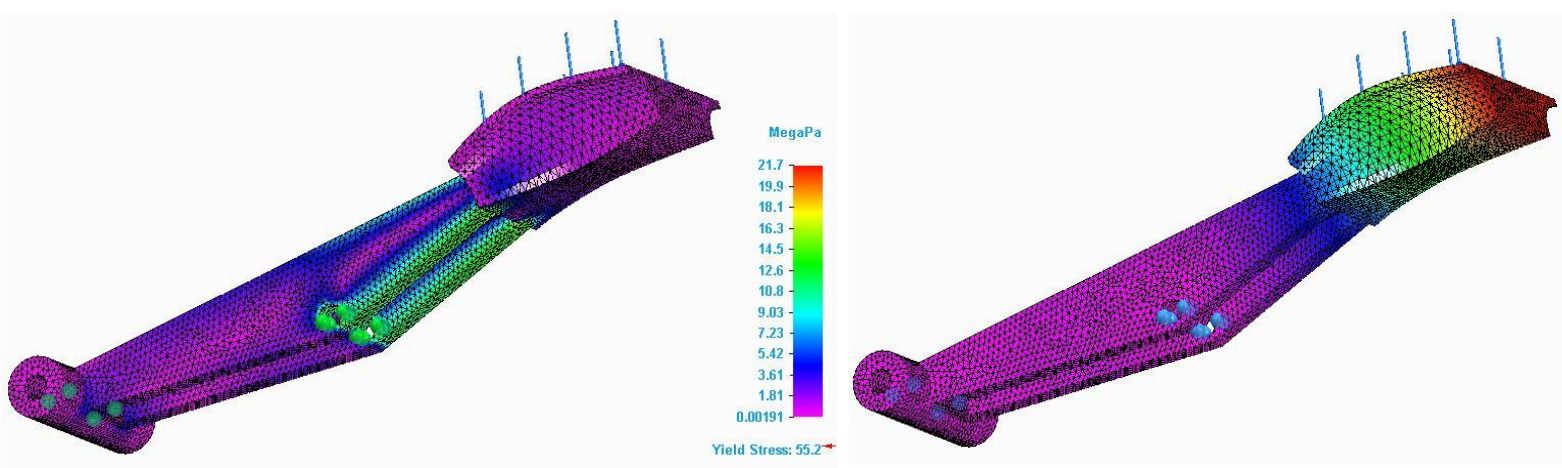

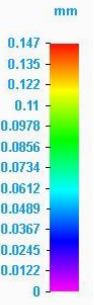

Figure 4. The Pedal Stress Distribution by Von Mises criteria (Left) and displacement distribution by Total Displacement criteria (Right)

The results of the simulation run were that $21.7 \mathrm{MPa}$ was the maximum stress against the yield strength of $55.158 \mathrm{MPa}$ which meant the factor of safety of this designed pedal was 2.54. The stress concentration zones can be seen in red colour at the ribs under the pedal face.

The objective of the finite element analysis was to identify the stress and displacement distribution in the pedal. Furthermore, it was essential to identify the factor of safety based on the maximum stress and the yield stress of material used. Based on the results shown above the factor of safety achieved was more than 2 .

The third step was an implementation of the generative design approach, As previously discussed, the generative design approach allows an exploration of a broad range of different design solutions for the initial model, therefore, the process is iterative to find the best solution that meets the requirements in terms of cost, applicability, and aesthetics. In this case, the initial design of the pedal is highlighted in Figure 1 and several generative design runs have been performed on it with two approaches, the first is to change the mass reduction ratio after each successful run, the second is to increase the quality of the study after each run, which consequently increases the processing time. The studies were conducted while maintaining a set of constraints and other parameters fixed such as safety factor, applied load, and preserved region. The safety factor was set to 1.5, and the applied force is of $450 \mathrm{~N}$ magnitude as shown in Figure 2.

In Figure 5, the quality of the study was set to 60 minutes long, which is adequate for the study considering the available computational capacity. 


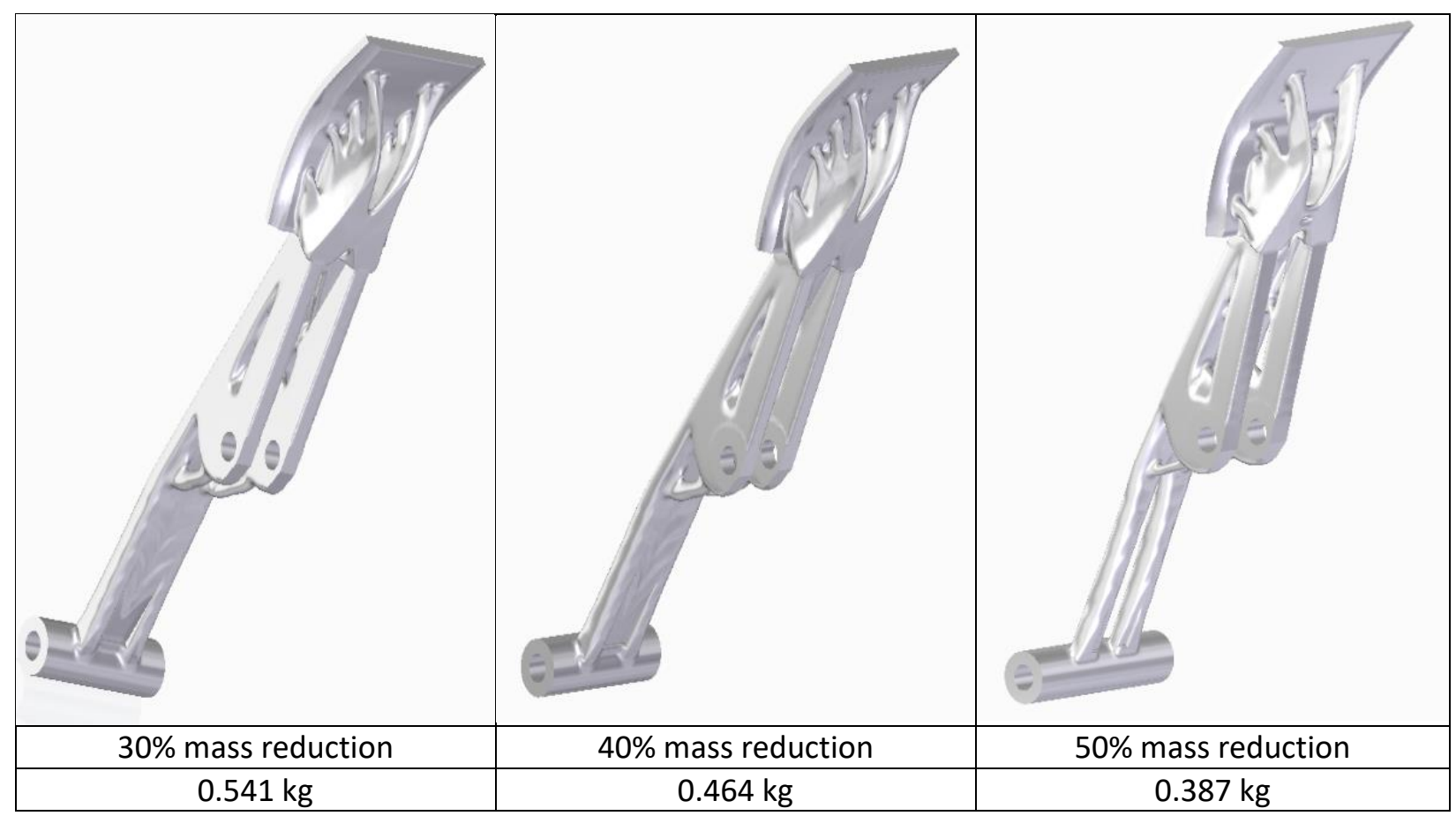

Figure 5. Generative Design Runs at Different Mass Reduction Ratios, Initial Mass $0.773 \mathrm{~kg}$

In Figure 6, the mass reduction ratio was set to $40 \%$ at a target mass of $=0.464 \mathrm{~kg}$ and the quality of the study was changing after each run, other parameters are fixed.

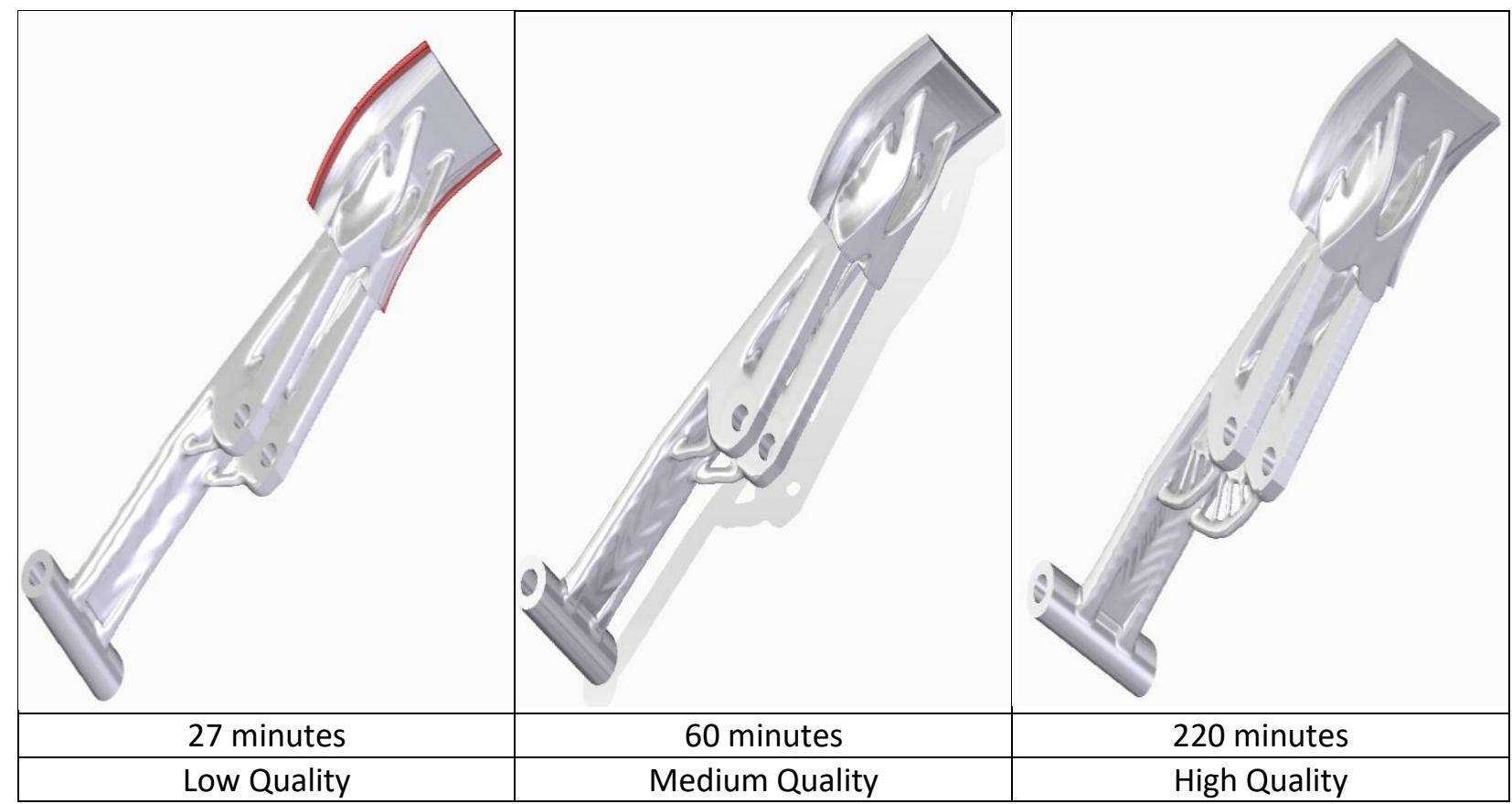

Figure 6. Generative Design Runs at Different Study Qualities with processing times

The objective of the comparative analysis of the different design parameters of the generative design was to identify the best parameters that will generate a robust design with high percentage of mass reduction and moderately complex design. It was deduced from the above comparison that there were no major changes below 60 minutes, however, as the quality increases, the required computational time increases and results in a robust, yet complex design compared to the initial model. 
The fourth step was to investigate the conventional manufacturing methods for mechanical pedals and compare the finite element analysis of these to the original designed pedal. The most common methods of manufacturing the mechanical pedal are either welding or forging. To this end, the mechanical pedal model that are based on the conventional methods of manufacturing such as welding, or forging are given in the Figures below.
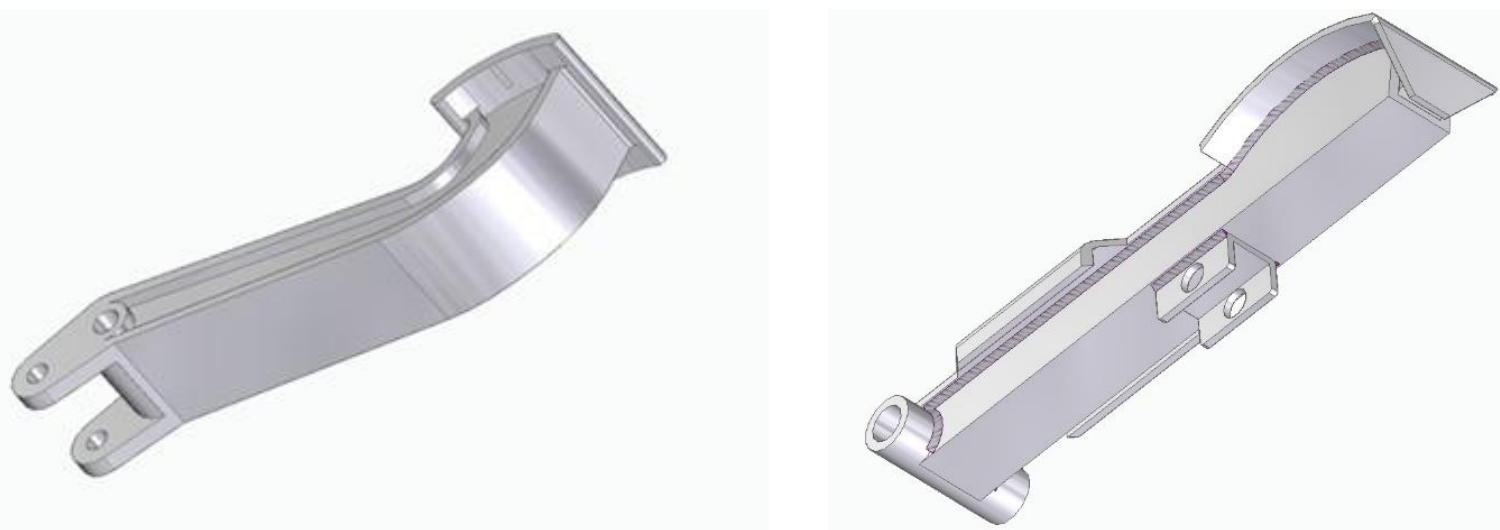

Figure 7. Redesign of a forged (left) and welded (right) mechanical pedal

Furthermore, the redesign of the above parts (forged and welded) was done again, keeping in view the key features of the generative designs that were previously conducted. These key features highlighted by the generative designs aimed to reduce the mass of the original pedal design. These key design features were incorporated in the new pedal designs that can be manufactured by the conventional methods such as forging, casting and welding. This is especially useful keeping in mind the costs and complexity associated with the use of additive manufacturing for the designs suggested by the generative design runs of solid edge. Given below in Figure 8 and 9 are the conceptual redesigned pedals that have the incorporated features of the generative designs with the reduced mass and appropriate factor of safety with conventional manufacturing methods in mind.

The first pedal redesign (concept redesign 1) had a mass of $0.470 \mathrm{~kg}$ (39\% mass reduction) and factor of safety value of 1.59, which was based on the manufacturing process of casting and welding. This design is indicated in the Figure 8 along with its respective stress distribution profile.
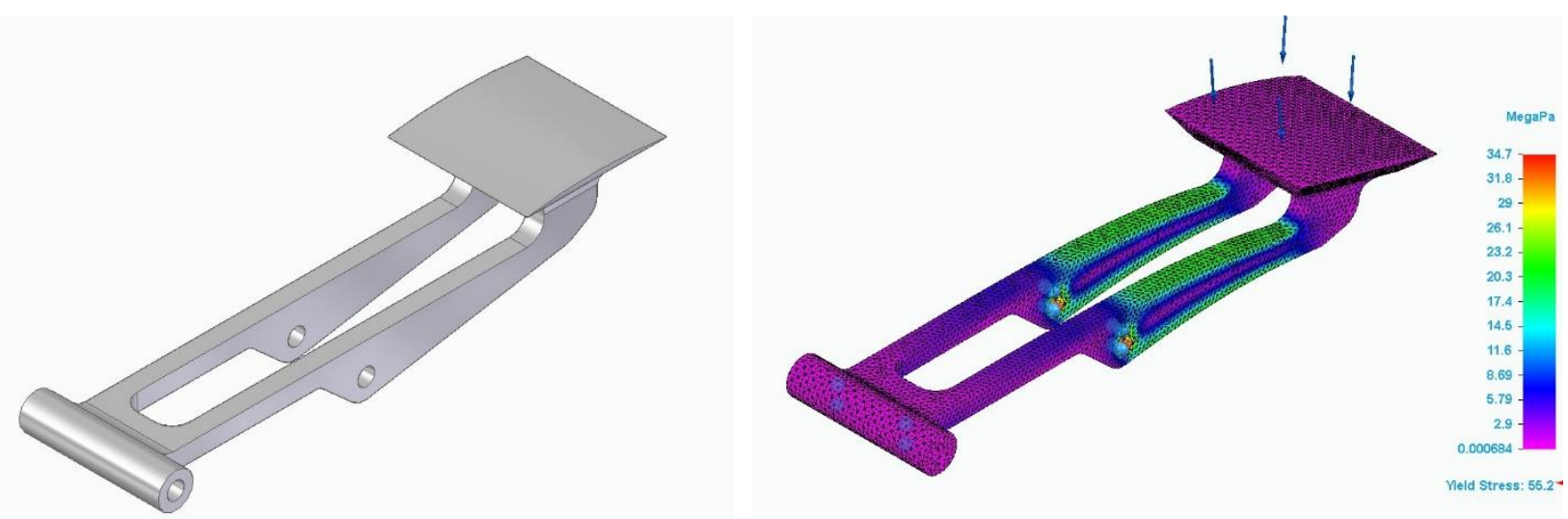

Figure 8. Concept redesign 1 with the stress distribution 
The second pedal redesign (concept redesign 2) had a mass of $0.458 \mathrm{~kg}$ ( $40 \%$ mass reduction) and factor of safety value of 1.63 , which was based on the manufacturing process of casting, and welding. This design is indicated in the Figure 9 along with its respective stress distribution profile.
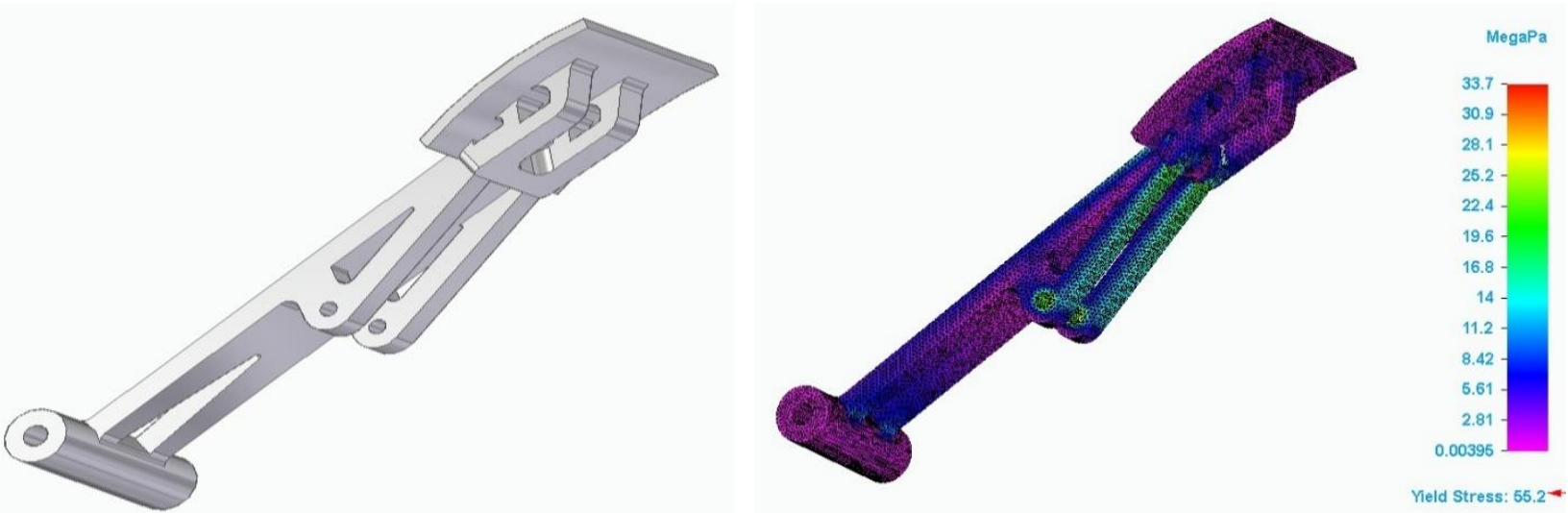

Figure 9. Concept redesign 2 and the stress distribution

\section{Conclusions}

The main objective was to redesign the mechanical pedal such that the mass of the pedal is reduced by using generative design as a guidance tool. First the mesh sensitivity analysis and finite element analysis of a standard pedal design, using the material as Al-5050, were conducted to help identify the appropriate mesh size and factor of safety for the model. Then generative designs for the mechanical pedal were conducted using specific design parameters such as percentage mass reduction, required factor of safety and an appropriate quality. The resulting generative design runs highlighted the key features in the pedal design that would help to reduce the mass by about $40 \%$ and maintain a factor of safety of approximately 1.5 . These key features were incorporated in redesigned pedal models, while keeping in mind the conventional methods of manufacturing such as casting, forging, welding, and drawing. These redesigned parts were then analysed using finite element analysis to avoid any stress concentrations and fractures while keeping all the stresses in the allowable region. The finite element analysis of the redesigned mechanical pedal helps to shortlist the design which has the highest factor of safety with maximum mass reduction. This design can be chosen as the most appropriate model for manufacturing mechanical pedals that are light weight, mechanically strong and robust in nature. 


\section{References}

[1] J. M. Snyder, J. T. Kajiya, and H. B. Jovanovicli, Generative Modeling For Symbolic Shape Design Using Interval Analysis. 1992.

[2] H. Li and R. Lachmayer, "Automated exploration of design solution space applying the generative design approach," Proc. Int. Conf. Eng. Des. ICED, vol. 2019-Augus, no. August, pp. 1085-1094, 2019, doi: 10.1017/dsi.2019.114.

[3] S. G. Shina, Concurrent Engineering and Design for Manufacture of Electronics Products. 1991.

[4] M. McKnight, "Generative Design: What it is? How is it being used? Why it's a game changer," KnE Eng., vol. 2, no. 2, pp. 176-181, 2017, doi: 10.18502/keg.v2i2.612.

[5] D. Vlah, R. Žavbi, and N. Vukašinović, "Evaluation of Topology Optimization and Generative Design Tools As Support for Conceptual Design," Proc. Des. Soc. Des. Conf., vol. 1, pp. 451-460, 2020, doi: $10.1017 /$ dsd.2020.165.

[6] J. GAVAČOVÁ, M. VEREŠ, and M. GRZNÁR, “COMPUTER AIDED GENERATIVE DESIGN OF AUTOMOTIVE SHAPED COMPONENTS," ACTA Teh. CORVINIENSIS - Bull. Eng., pp. 19-22, 2014.

[7] J. Gulanová, L. Gulan, M. Forrai, and M. Hirz, "Generative engineering design methodology used for the development of surface-based components," Comput. Aided. Des. Appl., vol. 14, no. 5, pp. 642-649, 2017, doi: 10.1080/16864360.2016.1273581.

[8] A. Peñaherrera, "Generative Design Optimization Process for Developing an E-bike Frame Using SolidThinking Inspire,” UNIVERSIDAD SAN FRANCISCO DE QUITO, 2019.

[9] A. Joakim, "DESIGN OPTIMIZATION OF A CONNECTING ROD FOR INTERNAL COMBUSTION ENGINE Presented to the Department of Mechanical and Aerospace Engineering California State University , Long Beach In Partial Fulfillment of the Requirements for the Degree Master of Science," California State University, 2020.

[10] S. Bagassi, F. Lucchi, F. De Crescenzio, and F. Persiani, "Generative design: Advanced design optimization processes for aeronautical applications," 30th Congr. Int. Counc. Aeronaut. Sci. ICAS 2016, pp. 1-7, 2016.

[11] S. Khan, E. Gunpinar, and B. Sener, "GenYacht: An interactive generative design system for computer-aided yacht hull design," Ocean Eng., vol. 191, pp. 1-16, 2019, doi: 10.1016/j.oceaneng.2019.106462.

[12] D. Huri and T. Mankovits, "Automotive rubber part design using machine learning," IOP Conf. Ser. Mater. Sci. Eng., vol. 659, no. 1, 2019, doi: 10.1088/1757-899X/659/1/012022.

[13] D. Huri and T. Mankovits, "Parameter selection of local search algorithm for design optimization of automotive rubber bumper," Appl. Sci., vol. 10, no. 10, 2020, doi: 10.3390/app10103584.

[14] Giraud-Moreau, Laurence, and Pascal Lafon. "A comparison of evolutionary algorithms for mechanical design components." Engineering Optimization 34.3 (2002): 307-322. 
[15] Krish, Sivam. "A practical generative design method." Computer-Aided Design 43.1 (2011): 88100.

[16] Toptas, Ersin. "Innovative approach to the design of mechanical parts." Journal of Mechatronics and Artificial Intelligence in Engineering 1.1 (2020): 14-20.

[17] Mačužić, Slavica, et al. "Pedal force determination respect to ride comfort." IOP Conference Series: Materials Science and Engineering. Vol. 252. No. 1. IOP Publishing, 2017. 